\title{
Chitin distribution in the Oithona digestive and reproductive systems revealed by fluorescence microscopy
}

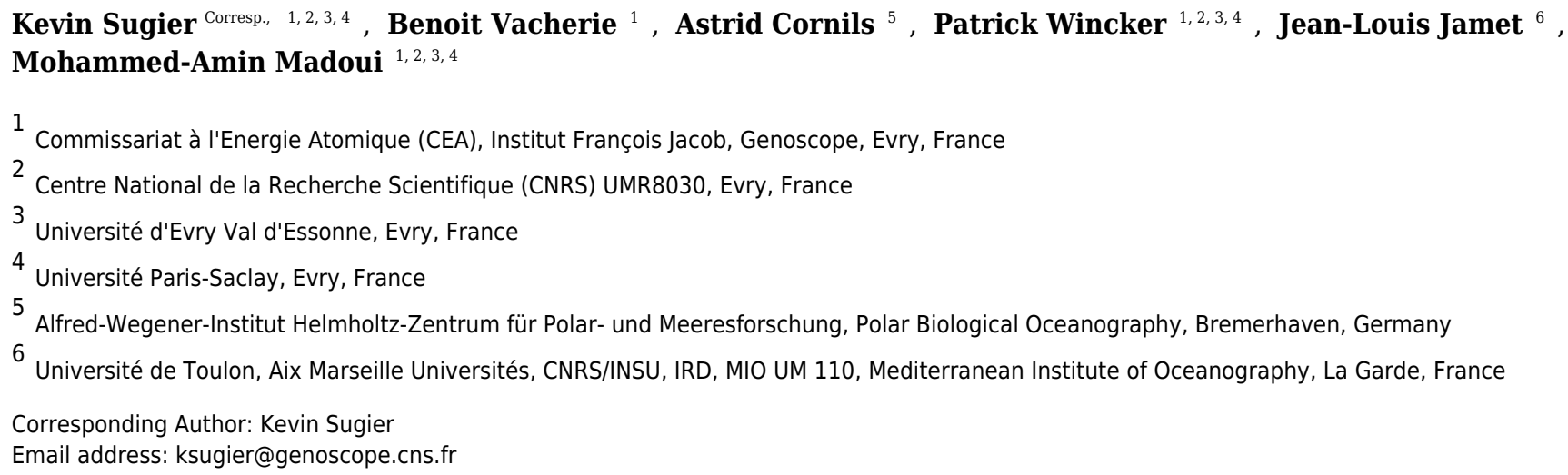

Among copepods, which are the most abundant animals on Earth, the genus Oithona is described as one of the most numerous and plays a major role in the marine food chain and biogeochemical cycles, particularly through the excretion of chitin-coated fecal pellets. Despite the morphology of several Oithona species is well known, knowledge of its internal anatomy and chitin distribution is still limited. To answer this problem, Oithona nana and Oithona similis individuals were stained by WGA-FITC and DAPI for fluorescence microscopy observations. The image analyses allowed a new description of the organization and chitin content of the digestive and reproductive systems of Oithona male and female. Chitin microfibrils were found all along the digestive system from the stomach to the hindgut with a higher concentration at the peritrophic membrane of the anterior midgut. Several midgut shrinkages were observed and proposed to be involved in fecal pellet shaping and motion. Amorphous chitin structures were also found to be a major component of the ducts and seminal vesicles and receptacles. The rapid staining protocol we proposed allowed a new insight into the Oithona internal anatomy and highlighted the role of chitin in the digestion and reproduction. This method could be applied to a wide range of copepods in order to perform comparative anatomy analyses. 
3 Chitin Distribution in the Oithona Digestive and Reproductive 4 Systems Revealed by Fluorescence Microscopy

7 Kevin Sugier ${ }^{1,2,3,4}$, Benoit Vacherie ${ }^{1}$, Astrid Cornils ${ }^{5}$, Jean-Louis Jamet ${ }^{6}$, Patrick Wincker ${ }^{1,2,3,4}$,

8 Mohammed-Amin Madoui 1,2,3,4

$10{ }^{1}$ Commissariat à l'Energie Atomique (CEA), Institut François Jacob, Genoscope, Evry, France

$11{ }^{2}$ Centre National de la Recherche Scientifique (CNRS) UMR8030, Evry, France

12 3 Université d'Evry Val d'Essonne, 2 rue Gaston Crémieux, 91057 Evry, France

$13{ }^{4}$ Université Paris-Saclay, Evry, France

14 5.Alfred-Wegener-Institut Helmholtz-Zentrum für Polar- und Meeresforschung, Polar Biological

15 Oceanography, Bremerhaven, Germany

16 6. Université de Toulon, Aix Marseille Universités, CNRS/INSU, IRD, MIO UM 110, Mediterranean

17 Institute of Oceanography, La Garde, France. 


\section{Abstract}

21 Among copepods, which are the most abundant animals on Earth, the genus Oithona is described

22 as one of the most numerous and plays a major role in the marine food chain and biogeochemical

23 cycles, particularly through the excretion of chitin-coated faecal pellets. Despite the morphology

24 of several Oithona species is well known, knowledge of its internal anatomy and chitin

25 distribution is still limited. To answer this problem, Oithona nana and Oithona similis

26 individuals were stained by WGA-FITC and DAPI for fluorescence microscopy observations.

27 The image analyses allowed a new description of the organization and chitin content of the digestive and reproductive systems of Oithona male and female. Chitin microfibrils were found all along the digestive system from the stomach to the hindgut with a higher concentration at the peritrophic membrane of the anterior midgut. Several midgut shrinkages were observed and proposed to be involved in faecal pellet shaping and motion. Amorphous chitin structures were

32 also found to be a major component of the ducts and seminal vesicles and receptacles. The rapid staining protocol we proposed allowed a new insight into the Oithona internal anatomy and highlighted the role of chitin in the digestion and reproduction. This method could be applied to 35 a wide range of copepods in order to perform comparative anatomy analyses. 


\section{Introduction}

37 Copepods are the most abundant animals on Earth ahead of insects and nematodes (Humes 1994)

38 and inhabit all aquatic niches: groundwater, vernal ponds, glaciers, lakes, rivers and oceans

39 (Huys \& Boxshall 1991). Among marine copepods, Oithona has been described as the most

40 important marine planktonic genus in terms of abundance (Gallienne \& Robins 2001). A recent

41 study, based on the Tara Oceans metagenomic data, has shown the global distribution of Oithona

42 in coastal and open ocean waters (Madoui et al. 2017), which highlighted its key role as a major

43 secondary producer of the marine food chain (Beaugrand et al. 2003; Zamora-Terol et al. 2014).

44 The important contribution of copepods in the biological carbon pump has also been

45 demonstrated (Jonasdottir et al. 2015), in particular through the excretion of faecal pellets

46 (Steinberg \& Landry 2017) that sink, provide organic and inorganic compounds to

47 microplankton (Steinberg et al. 2002; Valdés et al. 2017), and deposit on the sediments where

48 they could remain as fossils for several thousand years (Bathmann et al. 1987; Haberyan 1985).

49 The biochemical analysis of the copepod faecal pellets has revealed a high amount of chitin

50 (Kirchner 1995), a beta-1-4 N-acetylglucosamine polymer, the most abundant biopolymer in

51 nature after celluloses (Kirchner 1995), and mostly known in copepods as a component of the

52 exoskeleton. Besides the role of copepods in the carbon pump, the abundance of chitin in the

53 faecal pellets also points out the implication of copepods in the global nitrogen cycle (Frangoulis

54 et al. 2004).

55 Morphological traits of more than forty Oithona species are well known (Razouls et al. 2005-

56 2018), especially the structure of the antennules, the oral appendages, the swimming legs and the

57 caudal rami (Nishida 1985). However, such morphological traits are only accessible through

58 finical dissections under the microscope that need expertise and are time-consuming. Recently, 
59 molecular tools have proven their usefulness in species identification (Cornils et al. 2017;

60 Madoui et al. 2017).

61 The detailed external anatomy of copepods has been analyzed through Congo red fluorescence

62 (Michels \& Büntzow 2010) and through electron microscopy that allowed the species

63 identification of copepods and the characterization of their external structures (Chang 2013;

64 Cuoc et al. 1997; Marques et al. 2017). Using an electron microscope, Oithona nana Giesbrecht,

651892 female sexual orifices with attached male spermatophores were observable (Huys \&

66 Boxshall 1991). Diagrams of marine and freshwater cyclopoids, which provide the structures of

67 the reproductive and digestive systems (Borradaile \& Potts 1935; Dussart \& Defaye 2001;

68 Kellogg 1902) were available. Some studies proposed methods to observe the reproductive

69 system of aldehyde-preserved copepods by direct light microscopy observation of individuals

70 (Eisfeld \& Niehoff 2007; Niehoff 2003; Niehoff \& Hirche 1996; Tande \& Hopkins 1981), by

71 staining of gonad with borax carmine (Tande \& Gronvik 1983; Tande \& Hopkins 1981), with

72 fluorescent polyunsaturated aldehydes (PUAs) probes (Wolfram et al. 2014), or with Fast Green

73 (Batchelder 1986). The internal anatomy of Oithona similis Claus, 1863 has been recently

74 described using phase contrast microscopy and provided the first insight into the organization of

75 the Oithona female reproductive system (Mironova \& Pasternak 2017). In the Wolfram et al

76 study, some pictures of the calanoid Acartia tonsa obtained using fluorescent PUA probes, also

77 allowed to determine the anatomy of the digestive system. Other studies (Bautista \& Harris

78 1992; Debes et al. 2008) used the chlorophyll fluorescence to determine the ingestion rates and

79 the gut contents, but without providing a clear structure of the digestive organs. Electron

80 microscopy revealed that chitin microfibrils are present in the anterior and posterior midgut 
81 peritrophic membrane (PM) of free-living and in the posterior PM of parasitic copepods

82 (Yoshikoshi \& Kô 1988), but no Oithona species have been included in the study.

83 For a better understanding of the ecological success of Oithona, a detailed knowledge of its

84 internal anatomy is crucial. Fluorescence microscopy based on a double staining coupling Wheat

85 Gamma Agglutinin-Fluorescein IsoThioCyanate (WGA-FITC) and DiAmidino-2-PhenylIndole

86 (DAPI) were used to elucidate the internal anatomy. DAPI is a blue fluorescent protein which

87 has an affinity to two nucleoids: adenine and thyrosin (Lin et al. 1977). This staining is widely

88 used to detect DNA in eukaryotes, prokaryotes and some viruses, without tissue-specificity.

89 FITC is a green fluorescent protein that can be conjugated with a wheat lectin that has an affinity

90 and specificity to $\mathrm{N}$-acetyl- $\beta$-D-glucosamine (Allen et al. 1973). WGA-FITC staining is widely

91 used for chitin detection by fluorescence, in a liquid medium containing lysed cells or directly on

92 whole organisms (El Gueddari et al. 2002; Farnesi et al. 2015; Fones et al. 2016; Godoy et al.

93 2015). On copepods, WGA-FITC was used only once; but after dissolution of the soft tissues

94 which did not allow the investigation of the internal anatomy (Mravec et al. 2014). In the present

95 study, we used WGA-FITC and DAPI staining to provide a new insight into the internal anatomy

96 and chitin content of Oithona nana and Oithona similis with a focus on their digestive and

97 reproductive systems.

98 Material and methods

Biological materials samples

Oithona nana and $O$. similis specimens were sampled at two locations of the Toulon harbor,

101 France, at the East of the little harbor of Toulon (Lat $43^{\circ} 06^{\prime} 52.1^{\prime \prime} \mathrm{N}$ and Long $05^{\circ} 55^{\prime} 42.7^{\prime \prime} \mathrm{E}$ )

102 and the North of the great harbor of Toulon (Lat $43^{\circ} 06^{\prime} 02.3^{\prime \prime} \mathrm{N}$ and Long $05^{\circ} 56^{\prime} 53.4^{\prime \prime} \mathrm{E}$ ). 
103 Sampling took place in November 2016, January, March and June 2017. The samples were

104 collected from the upper water layers $(0-10 \mathrm{~m})$ using zooplankton nets with a mesh of $90 \mu \mathrm{m}$ and

$105200 \mu \mathrm{m}$. Samples were preserved in $70 \%$ ethanol and stored at $-4^{\circ} \mathrm{C}$. In the samples, individuals

106 of the four different development stages were observable (nauplii, copepodites and adults of both

107 sexes), but the large majority were female adults.

108

109

110

111

112

113

114

115

116

117

118

119

120

121

122

123

124

\section{Individual staining}

This protocol was adapted from Farnesi et al. After gently mixing the ethanol preserved samples (about 20 reversals), $100 \mu \mathrm{L}$ were sampled in a $1.5 \mathrm{~mL}$ tube. After two minutes, the ethanol was removed, and $100 \mu \mathrm{L}$ of Phosphate Buffered Saline (PBS) at $1 \mathrm{X}$ and $10 \mu \mathrm{L}$ of WGA-FITC at $2 \mathrm{mg}$ $\mathrm{mL}^{-1}$ (Sigmaaldrich.com 2017b) were added for chitin staining. After mixing, the sample was incubated for 30 minutes protected from light before supernatant removing. To stain the DNA, dual staining with DAPI can be performed by adding, $100 \mu \mathrm{L}$ of PBS at $1 \mathrm{X}$ and $10 \mu \mathrm{L}$ of DAPI (Sigmaaldrich.com 2017a) at 10X. The microscopy observations were done directly after mixing. This protocol can also be used on living individuals from a seawater sample; in this case, sodium chloride at $39 \mathrm{~g} \mathrm{~L}^{-1}$ has to be added to the PBS solution.

\section{Microscopy}

The stained individuals were placed between slide and coverslip and observed under a reflected fluorescence microscope Olympus BX43. WGA-FITC was excited with the 460/495nm line from a 100W mercury lamp with an interference excitation filter (BP460), and collected with a 505nm dichroic mirror (DM505) and a 510nm interference barrier filter (BA510IF). DAPI fluorescence was excited with the 340/390nm line from a $100 \mathrm{~W}$ mercury lamp with an interference excitation filter (BP340), and collected with a 410nm dichroic mirror (DM410) and 
125 a 420nm interference barrier filter (BA420IF). Selected Oithona individuals were photographed

126 with a sixteen-megapixel camera using the ToupView software (v.3.7). For each individual, three

127 photographs were taken: one in polarized light, one with the WGA-FITC fluorescence and one

128 with the DAPI fluorescence. Some colour adjustments were made with the ImageJ software

129 (Schneider et al. 2012).

\section{Results}

\section{Oithona morphology with WGA-FITC microscopy}

132 The Oithona chitin was labelled with WGA-FITC directly on the individuals and observed by

133 fluorescence microscopy. The setae and spines of the exopod segments of the five leg pairs could

134 be identified and counted on $O$. nana (Figure 1.A). These first results revealed the chitinous

135 structure of the setae and the spines, and could provide a rapid method for taxonomical

136 identification. However, because of the individuals and setae position on the plate, we were not

137 able to identify and count the setae of all tested individuals. Chitinous elliptic or spherical

138 structures of unknown function and larger than 6 micrometres (Figure 1.A) were also visible in

139 the exopods of the swimming legs. These globular structures were observed in both sexes of

140 O. nana (Figure 1.A, 2.A, 2.B, 2.C, 2.D, 2.E), but only in female individuals of $O$. similis (Figure

141 2.F, 2.G). They may also be smaller (2.F), or absent (Figure 3.A, 4.C) in other individuals. These

142 structures can also be present in other exopod segments (Figure 2.A). Another tubular structure,

143 in the distal part of the exopods 3 of the right third leg, right and left fourth legs and right and left

144 fifth legs were noticeable (Figure 1.A). In other Oithona individuals, these tubular structures

145 appear to be attached to the globular structure (Figure 2.B, 2.D, 2.G).

\section{Chitin distribution in the Oithona digestive system}


147 Chitin was detected all along the digestive system, from the stomach to the hindgut of the 148 nauplius (Figure 1.B) and adults (Figure 1.C, 1.D, 1.E) of the two species. The exoskeleton chitin 149 was also stained by the WGA-FITC, which allowed a clear identification of the stomach in the 150 prosome, of the midgut in the prosome and in the urosome and the hindgut in the urosome. 151 Along the digestive system, the chitin had a microfibrillar structure aligned along the antero152 posterior axis with regions showing higher microfibrils density, especially the anterior midgut 153 and some stomach areas (Figure 1.C, 1.D, 1.E, 1.F). Some individuals contained in their anterior and posterior midgut one or several elliptical faecal pellets completely engulfed by chitin (Figure 1.E, 4.B). However, no faecal pellets were found in the nauplius. In the anterior and posterior midgut, we observed several shrinkages at different interval distances corresponding to midgut contractions. In certain cases, several shrinkages (up to four) were separated by less than five micrometres (Figure 1.D), while other individuals showed more distant shrinkages (Figure 1.F).

\section{Chitin distribution in the Oithona reproductive system}

The DAPI and WGA-FITC stainings on Oithona females allowed the identification of the ovaries and the oviducts that presented a heart shape in the middle of the prosome (Figure 3.A, 3.B) as previously described by Mironova \& Pasternak. The oviducts start from each lateral side of the gonads to the seminal receptacle in the genital double somite (the first two segments of the urosome). Comparing to the microfibrillar structure of the chitin found in the digestive system, the chitin staining in the reproductive system was mainly amorphous. Besides, its distribution was discontinuous along the ducts, altering chitin-rich and poor areas (Figure 3.C, 3.D).

In females, we distinguished two parts forming the seminal receptacle (Figure 3.E, 3.F). The first part was chitin-rich and located in the anterior region of the receptacle. The chitin distribution 
169 between the anterior receptacle and the oviduct was discontinuous. The second part was located

170 in the posterior receptacle and contained less and sparser chitin, presenting a mix of

171 microfibrillar and amorphous structures. Thanks to DAPI staining, in some females, the presence

172 of the DNA rich material in the posterior region of the seminal receptacle was observed and was

173 likely to be male semen.

174 In males, the chitin staining allowed the identification of the spermiducts, which presented the 175 same chitin pattern observed in the oviducts (Figure 4.A, 4.C). The spermiducts probably start 176 from each side of the male gonads (not visible on the pictures) to the seminal vesicles, in the 177 sexual somite (Figure 4.B). As for the female seminal receptacle, the male seminal vesicle can be 178 divided into two parts (Figure 4.D, 4.E). The first part of the vesicles is chitin-rich, located in the anterior region of the vesicle. The distribution of the chitin from this upper part of the vesicle to 180 the spermiduct was not continuous. The second part, located in the posterior region of the 181 vesicle, was observed by DAPI staining and was likely to be filled by DNA-rich male semen.

\section{Discussion}

183 Comparing to previous staining methods used to observe the digestive and reproductive systems 184 of copepods (Batchelder 1986; Eisfeld \& Niehoff 2007; Mironova \& Pasternak 2017; Niehoff 185 2003; Niehoff \& Hirche 1996; Tande \& Gronvik 1983; Tande \& Hopkins 1981; Wolfram et al. 186 2014), the protocol proposed here allows a clear insight into the chitin distribution in these 187 systems. Moreover, this protocol is simple and rapid, taking a few minutes of manipulation, 188 thirty minutes of incubation, and can be used on living, but also on alcohol-preserved copepods.

189 The main limit of our method remains in the short-time staining of the WGA-FITC: a picture 190 must be taken, a few minutes after fluorescence excitation to save any microscopic observation 
191 without loss of quality. Furthermore, for the reproductive system, the DAPI staining allows only

192 the observation of the gonad structure; while the Mironova and Pasternak protocol allows a

193 better identification of the oocytes.

194 The use of WGA-FITC revealed chitinous spherical structures in the exopods of the swimming

195 legs in O. nana males and females and in $O$. similis females, which were not observed in

196 previous studies. The absence of these structures in $O$. similis male individuals may be a bias due

197 to their low presence in our samples. Despite, luminescence is not conspicuous in Oithona, these

198 structures could be luminous glands (Herring 1988). The green staining revealed also a chitinous

199 tubular structure in the exopods penultimate segment of the swimming legs. These structures

200 resemble the 'Crusalis organ', an osmoregulatory structure that was described by Johnson et al in

2012014 from the coastal/estuarine copepod Eurytemora affinis. Since the globular and the tubular

202 structures seemed attached, we suggest they form only one organ involved either in

203 bioluminescence, osmoregulation or both.

204 The WGA-FITC staining allowed also the identification of the chitin distribution in the Oithona

205 organs, which provides a high-quality view of the external and internal anatomy and pointed out

206 the major role of chitin in the Oithona digestion and reproduction. According to insect studies,

207 the distribution of chitin in the digestive system is limited to the midgut (Hegedus et al. 2009;

208 Terra 2001). The same chitin distribution was observed in decapods (Martin et al. 2006; Wang et

209 al. 2012). In both Oithona species, we detected chitin throughout the digestive system, which

210 distinguishes it from insects and decapods, but seems consistent with observations in other free-

211 living cyclopoids made by Yoshikoshi and Kô, in 1988. 
212 In the PM of some insects (Hegedus et al. 2009; Kelkenberg et al. 2015; Lehane 1997), chitin

213 plays a role in protection (chemical, mechanical and against viruses, bacteria and pathogens) and

214 digestion (Terra 2001). As the synthesis of chitin has a significant metabolic cost for the

215 organism, we hypothesized that, like the insects and decapods PM, the formation of a chitin coat

216 around faecal pellets help to protect against toxins and pathogens that were not degraded during

217 digestion.

218 In copepods, no evidence of midgut contraction has previously been described although the 219 phenomenon has been suggested at several instances (Gauld 1957). We suppose that the midgut 220 shrinkages observed in this study could play a key role in the formation and motion of the faecal 221 pellets to the anus. However, we observed intestine shrinkages without the presence of faecal pellets, and vice versa. As proposed by Yoshikoshi and Kô for other copepods, we also suggest that, in Oithona, the formation of chitin coat around the faecal pellets can be produced by engulfing digested food in chitin microfibrils present in the PM of the anterior midgut (Figure 5).

The presence of chitin along the oviduct and spermiduct walls validates the cuticular appearance of the ducts described by Cuoc et al. In all Oithona males, we observed a pair of spermiducts, while in Calanus finmarchicus one of the two spermiducts disappeared during the male differentiation (Tande \& Hopkins 1981). The bipartite structure of the seminal receptacles and vesicles found in $O$. nana and $O$. similis males and females were very similar. In males, we hypothesized that the chitinous structure of the vesicle plays a role in the holding of the spermatophores during their formation. Likewise, in the females, this structure would play a role in the holding of the ovisac but also in the opening and closing of the oviduct to release oocytes 233 in the seminal receptacle. 


\section{Conclusion}

235 With this study, we adapted and tested a simple and rapid chitin-staining protocol that can help

236

237

238

239

240

241

242

243

244

to the taxonomic identification of copepods, and enable new studies on copepod comparative anatomy at a larger scale. The application of the method to Oithona extended the knowledge of the structure of its digestive and reproductive systems. Considering the important role of copepods in the carbon and nitrogen sequestration through chitin synthesis, more efforts should be undergone to better understand the molecular and physiological mechanisms involved in faecal pellets formation.

\section{Acknowledgements}

We thank Julie Poulain for initiating the Oithona genome project and Dr Leocadio BlancoBercial for helpful comments on the manuscript.

\section{References}

Allen AK, Neuberger A, and Sharon N. 1973. The purification, composition and specificity of wheat-germ agglutinin. Biochem $J$ 131:155-162.

Batchelder HP. 1986. A Staining Technique for Determining Copepod Gonad Maturation Application to Metridia-Pacifica from the Northeast Pacific-Ocean. Journal of Crustacean Biology 6:227-231. DOI 10.2307/1547983

Bathmann UV, Noji TT, Voss M, and Peinert R. 1987. Copepod Fecal Pellets - Abundance, Sedimentation and Content at a Permanent Station in the Norwegian Sea in May June 1986. Marine Ecology Progress Series 38:45-51. DOI 10.3354/Mepso38045

Bautista B, and Harris RP. 1992. Copepod Gut Contents, Ingestion Rates and Grazing Impact on Phytoplankton in Relation to Size Structure of Zooplankton and Phytoplankton during a Spring Bloom. Marine Ecology Progress Series 82:41-50. DOI 10.3354/Mepso82041

Beaugrand G, Brander KM, Alistair Lindley J, Souissi S, and Reid PC. 2003. Plankton effect on cod recruitment in the North Sea. Nature 426:661-664. DOI 10.1038/natureo2164

Borradaile LA, and Potts FA. 1935. Class Copepoda. The Invertebrata: a manual for the use of students. New York: The MacMillan Company, 370-376. 
261

262

263

264

265

266

267

268

269

270

271

272

273

274

275

276

277

278

279

280

281

282

283

284

285

286

287

288

289

290

291

292

293

294

295

296

297

Chang C. 2013. A New Species of Cletocamptus Copepoda (Harpacticoida, Canthocamptidae) from Salt Marshes in Korea.

Cornils A, Wend-Heckmann B, and Held C. 2017. Global phylogeography of Oithona similis s.l. (Crustacea, Copepoda, Oithonidae) - A cosmopolitan plankton species or a complex of cryptic lineages? Mol Phylogenet Evol 107:473-485. DOI 10.1016/j.ympev.2016.12.019

Cuoc C, Defaye D, Brunet M, Notonier R, and Mazza J. 1997. Female genital structures of Metridinidae (Copepoda: Calanoida). Marine Biology 129:651-665. DOI 10.1007/s002270050208

Debes H, Eliasen K, and Gaard E. 2008. Seasonal variability in copepod ingestion and egg production on the Faroe shelf. Hydrobiologia 600:247-265. DOI 10.1007/s10750-0079238-3

Dussart BH, and Defaye D. 2001. Introduction to the Copepoda. Leiden: Backhuys.

Eisfeld SM, and Niehoff B. 2007. Gonad morphology, oocyte development and spawning cycle of the calanoid copepod Acartia clausi. Helgoland Marine Research 61:193-201. DOI 10.1007/s10152-007-0066-7

El Gueddari NE, Rauchhaus U, Moerschbacher BM, and Deising HB. 2002. Developmentally regulated conversion of surface-exposed chitin to chitosan in cell walls of plant pathogenic fungi. New Phytologist 156:103-112. DOI 10.1046/j.1469-8137.2002.00487.x

Farnesi LC, Menna-Barreto RF, Martins AJ, Valle D, and Rezende GL. 2015. Physical features and chitin content of eggs from the mosquito vectors Aedes aegypti, Anopheles aquasalis and Culex quinquefasciatus: Connection with distinct levels of resistance to desiccation. J Insect Physiol 83:43-52. DOI 10.1016/j.jinsphys.2015.10.006

Fones HN, Mardon C, and Gurr SJ. 2016. A role for the asexual spores in infection of Fraxinus excelsior by the ash-dieback fungus Hymenoscyphus fraxineus. Sci Rep 6:34638. DOI $10.1038 /$ srep34638

Frangoulis C, Christou ED, and Hecq JH. 2004. Comparison of Marine Copepod Outfluxes: Nature, Rate, Fate and Role in the Carbon and Nitrogen Cycles. Adv Mar Biol: Academic Press, 253-309.

Gallienne CP, and Robins DB. 2001. Is Oithona the most important copepod in the world's oceans? Journal of Plankton Research 23:1421-1432. DOI 10.1093/plankt/23.12.1421

Gauld DT. 1957. A Peritrophic Membrane in Calanoid Copepods. Nature 179:325-326. DOI $10.1038 / 179325$ ao

Godoy RS, Fernandes KM, and Martins GF. 2015. Midgut of the non-hematophagous mosquito Toxorhynchites theobaldi (Diptera, Culicidae). Sci Rep 5:15836. DOI 10.1038/srep15836

Haberyan KA. 1985. The Role of Copepod Fecal Pellets in the Deposition of Diatoms in Lake Tanganyika. Limnology and Oceanography 30:1010-1023. DOI 10.4319/lo.1985.30.5.1010 
298

299

300

301

302

303

304

305

306

307

308

309

310

311

312

313

314

315

316

317

318

319

320

321

322

323

324

325

326

327

328

329

330

331

332

333

334

Hegedus D, Erlandson M, Gillott C, and Toprak U. 2009. New insights into peritrophic matrix synthesis, architecture, and function. Annu Rev Entomol 54:285-302. DOI 10.1146/annurev.ento.54.110807.090559

Herring PJ. 1988. Copepod Luminescence. Hydrobiologia 167:183-195. DOI 10.1007/Bfooo26304

Humes AG. 1994. How many copepods? Hydrobiologia 292:1-7. DOI 10.1007/BFo0229916

Huys R, and Boxshall GA. 1991. Copepod Evolution. London: The Ray Society.

Johnson KE, Perreau L, Charmantier G, Charmantier-Daures M, and Lee CE. 2014. Without gills: localization of osmoregulatory function in the copepod Eurytemora affinis. Physiological and Biochemical Zoology 87:310-324. DOI 10.1086/674319

Jonasdottir SH, Visser AW, Richardson K, and Heath MR. 2015. Seasonal copepod lipid pump promotes carbon sequestration in the deep North Atlantic. Proc Natl Acad Sci U S A 112:12122-12126. DOI 10.1073/pnas.1512110112

Kelkenberg M, Odman-Naresh J, Muthukrishnan S, and Merzendorfer H. 2015. Chitin is a necessary component to maintain the barrier function of the peritrophic matrix in the insect midgut. Insect Biochem Mol Biol 56:21-28. DOI 10.1016/j.ibmb.2014.11.005

Kellogg VL. 1902. Branch Arthropoda: the Crustaceans, Centipeds, Insects, and Spiders. Elementary Zoology. New York: Henry Holt \& Co., 148.

Kirchner M. 1995. Microbial Colonization of Copepod Body Surfaces and Chitin Degradation in the Sea. Helgoländer Meeresuntersuchungen 49:201-212. DOI 10.1007/Bfo2368350

Lehane MJ. 1997. Peritrophic matrix structure and function. Annu Rev Entomol 42:525-550. DOI 10.1146/annurev.ento.42.1.525

Lin MS, Comings DE, and Alfi OS. 1977. Optical Studies of the interaction of 4'-6'-diamidino-2phenylindole with DNA and metaphase chromosomes. Chromosoma 60:15-25.

Madoui MA, Poulain J, Sugier K, Wessner M, Noel B, Berline L, Labadie K, Cornils A, BlancoBercial L, Stemmann L, Jamet JL, and Wincker P. 2017. New insights into global biogeography, population structure and natural selection from the genome of the epipelagic copepod Oithona. Mol Ecol 26:4467-4482. DOI 10.1111/mec.14214

Marques TM, Clebsh L, Córdova L, and Boeger WA. 2017. Ergasilus turkayi n. sp. (Copepoda, Cyclopoida, Ergasilidae): a gill parasite of Serrasalmus hollandi Jégu, 2003 (Characiformes, Serrasalmidae) from the Paragùa River, Bolivia. Nauplius 25.

Martin GG, Simcox R, Nguyen A, and Chilingaryan A. 2006. Peritrophic membrane of the penaeid shrimp Sicyonia ingentis: structure, formation, and permeability. Biol Bull 211:275-285. DOI 10.2307/4134549

Michels J, and Büntzow M. 2010. Assessment of Congo red as a fluorescence marker for the exoskeleton of small crustaceans and the cuticle of polychaetes. $J$ Microsc 238:95-101. DOI 10.1111/j.1365-2818.2009.03360.x 
335

336

337

338

339

340

341

342

343

344

345

346

347

348

349

350

351

352

353

354

355

356

357

358

359

360

361

362

363

364

365

366

367

368

369

370

371

372

Mironova E, and Pasternak A. 2017. Female gonad morphology of small copepods Oithona similis and Microsetella norvegica. Polar Biology 40:685-696. DOI 10.1007/s00300016-1993-z

Mravec J, Kracun SK, Rydahl MG, Westereng B, Miart F, Clausen MH, Fangel JU, Daugaard M, Van Cutsem P, De Fine Licht HH, Hofte H, Malinovsky FG, Domozych DS, and Willats WG. 2014. Tracking developmentally regulated post-synthetic processing of homogalacturonan and chitin using reciprocal oligosaccharide probes. Development 141:4841-4850. DOI 10.1242/dev.113365

Niehoff B. 2003. Gonad morphology and oocyte development in Pseudocalanus spp. in relation to spawning activity. Marine Biology 143:759-768. DOI 10.1007/s00227-003-1034-7

Niehoff B, and Hirche HJ. 1996. Oogenesis and gonad maturation in the copepod Calanus finmarchicus and the prediction of egg production from preserved samples. Polar Biology 16:601-612.

Nishida S. 1985. Taxonomy and distribution of the family Oithonidae (Copepoda, Cyclopoida) in the Pacific and Indian Oceans. Tokyo: Ocean Research Institute, University of Tokyo.

Razouls C, de Bovée F, Kouwenberg J, and Desreumaux N. 2005-2018. Diversity and Geographic Distribution of Marine Planktonic Copepods. http://copepodesobsbanyulsfr/.

Schneider CA, Rasband WS, and Eliceiri KW. 2012. NIH Image to ImageJ: 25 years of image analysis. Nat Methods 9:671-675.

Sigmaaldrich.com. 2017a. DAPI for nucleic acid staining

Available at http://www.sigmaaldrich.com/catalog/product/sigma/d9542 (accessed 09/11 2017).

Sigmaaldrich.com. 2017b. Lectin from Triticum vulgaris (wheat) FITC conjugate, lyophilized powder. Available at http://www.sigmaaldrich.com/catalog/product/sigma/l4895 (accessed 09/11 2017).

Steinberg DK, Goldthwait SA, and Hansell DA. 2002. Zooplankton vertical migration and the active transport of dissolved organic and inorganic nitrogen in the Sargasso Sea. DeepSea Research Part I-Oceanographic Research Papers 49:1445-1461. DOI 10.1016/So967-0637(02)00037-7

Steinberg DK, and Landry MR. 2017. Zooplankton and the Ocean Carbon Cycle. Ann Rev Mar Sci 9:413-444. DOI 10.1146/annurev-marine-010814-015924

Tande KS, and Gronvik S. 1983. Ecological Investigations on the Zooplankton Community of Balsfjorden, Northern Norway - Sex-Ratio and Gonad Maturation Cycle in the Copepod Metridia-Longa (Lubbock). Journal of Experimental Marine Biology and Ecology 71:43-54. DOI 10.1016/0022-0981(83)90103-X

Tande KS, and Hopkins CCE. 1981. Ecological Investigations of the Zooplankton Community of Balsfjorden, Northern Norway - the Genital System in Calanus-Finmarchicus and the 
Role of Gonad Development in Overwintering Strategy. Marine Biology 63:159-164. DOI 10.1007/Bfoo406824

Terra WR. 2001. The origin and functions of the insect peritrophic membrane and peritrophic gel. Arch Insect Biochem Physiol 47:47-61. DOI 10.1002/arch.1036

Valdés VP, Fernandez C, Molina V, Escribano R, and Joux F. 2017. Dissolved Compounds Excreted by Copepods Reshape the Active Marine Bacterioplankton Community Composition. Frontiers in Marine Science 4. DOI 10.3389/fmars.2017.00343

Wang L, Li F, Wang B, and Xiang J. 2012. Structure and partial protein profiles of the peritrophic membrane (PM) from the gut of the shrimp Litopenaeus vannamei. Fish Shellfish Immunol 33:1285-1291. DOI 10.1016/j.fsi.2012.09.014

Wolfram S, Nejstgaard JC, and Pohnert G. 2014. Accumulation of polyunsaturated aldehydes in the gonads of the copepod Acartia tonsa revealed by tailored fluorescent probes. PLoS One 9:e112522. DOI 10.1371/journal.pone.0112522

Yoshikoshi K, and Kô Y. 1988. Structure and Function of the Peritrophic Membranes of Copepods. Nippon Suisan Gakkaishi 54:1077-1082. DOI 10.2331/suisan.54.1077

Zamora-Terol S, Kjellerup S, Swalethorp R, Saiz E, and Nielsen TG. 2014. Population dynamics and production of the small copepod Oithona spp. in a subarctic fjord of West Greenland. Polar Biology 37:953-965. DOI 10.1007/so0300-014-1493-y 


\section{Figure 1 (on next page)}

Oithona appendages morphology and digestive system by WGA-FITC fluorescence microscopy.

st: stomach, mg: midgut, hg: hindgut, sh: shrinkage, ex: exopod, se: setal, sp: spine, fp: fecal pellet, a: globular structure, $\mathbf{b}$ : tubular structure. A. Dorsal view of the $O$. nana female swimming appendages. B. Lateral view of the Oithona nauplius digestive system. C. Lateral view of the $O$. nana female stomach. D. Dorsal view of the $O$. nana female stomach. $\mathbf{E}$. Lateral view of an $O$. nana male gut. F. Dorsal view of an $O$. similis female adult stomach. 


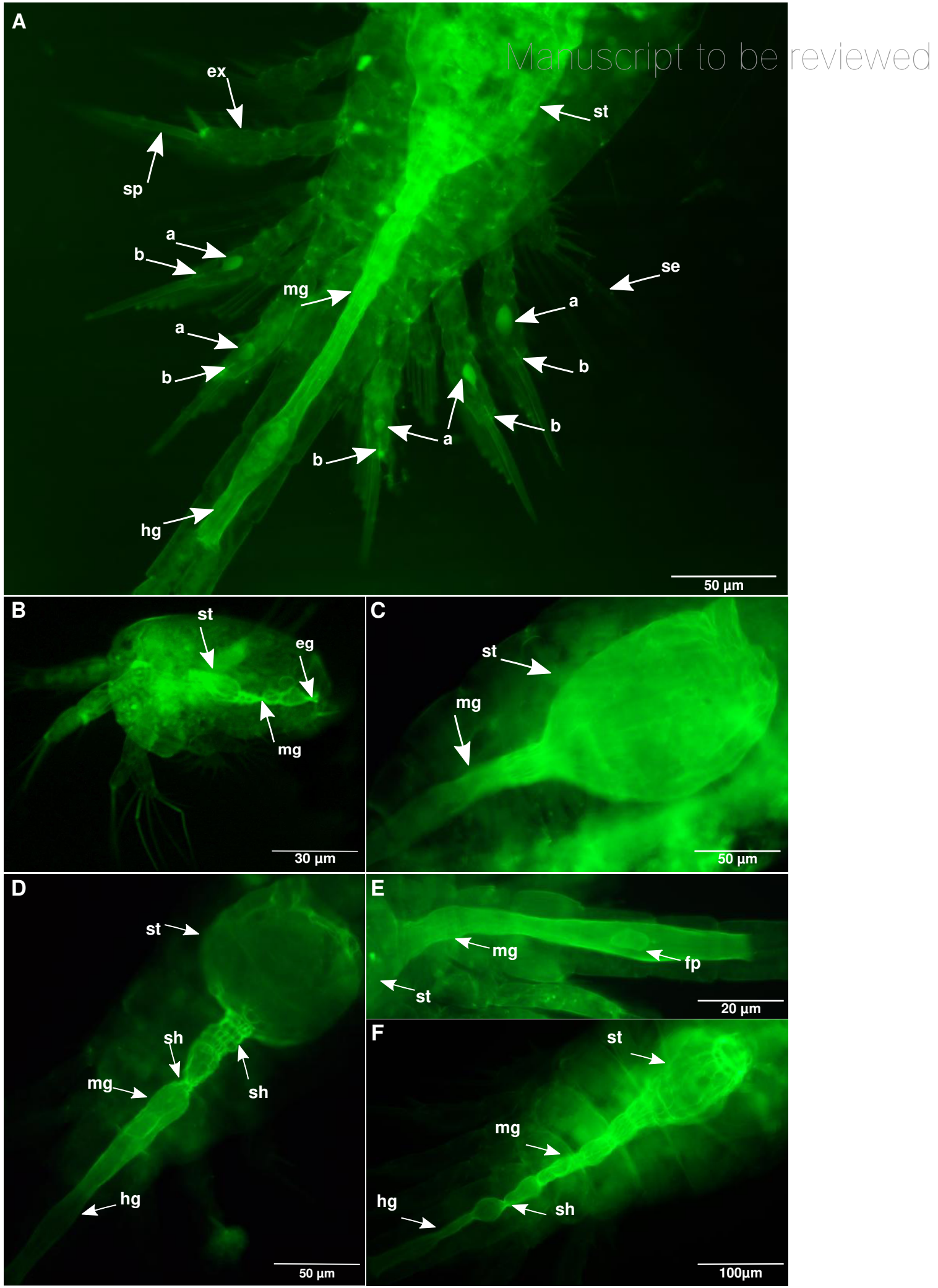




\section{Figure 2 (on next page)}

Oithona globular and tubular chitinous structures in the swimming appendages by WGAFITC fluorescence microscopy.

mg: midgut, sr: seminal receptacle, s: semen, ov: oviduct, hg: hindgut, b: diffuse chitin region, c: chitin rich region. A. Dorsal view of the $O$. nana female reproductive system. B. Dorsal view of the $O$. similis female reproductive system. C. Dorsal view of the $O$. nana female double sexual somite. 


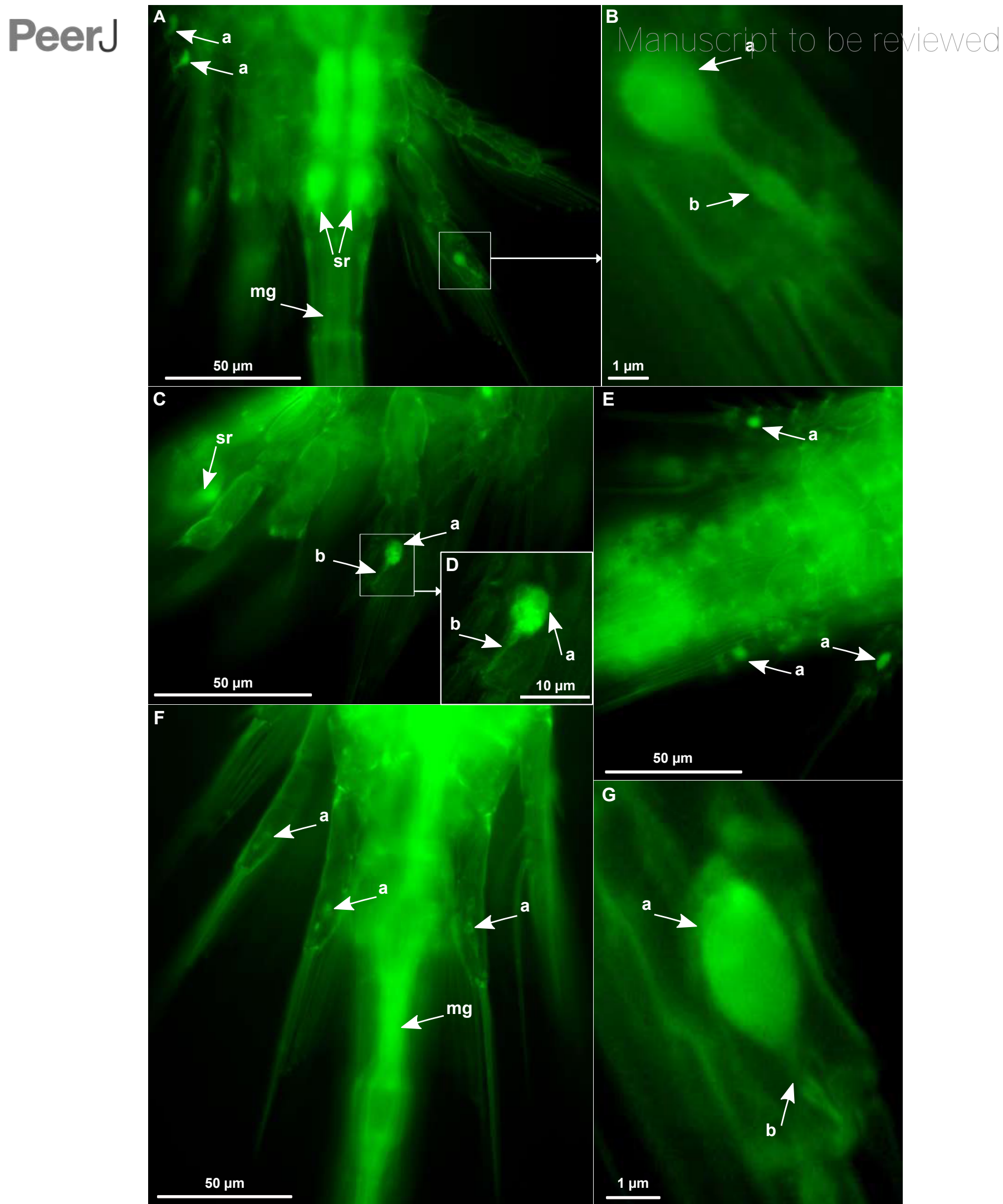




\section{Figure 3 (on next page)}

Oithona female reproductive system by DAPI and WGA-FITC fluorescence microscopy

mg: midgut, sr: seminal receptacle, s: semen, ov: oviduct, hg: hindgut, b: diffuse chitin region, c: chitin rich region. A-B.. Dorsal view of the O.nana female reproductive system (DAPI staining on the left and WGA-FITC staining on the right). C-D. Dorsal view of the $O$. similis female reproductive system (DAPI staining on the left and WGA-FITC staining on the right). E-F. Dorsal view of the $O$. nana female double sexual somite (DAPI staining on the left and WGA-FITC staining on the right). 

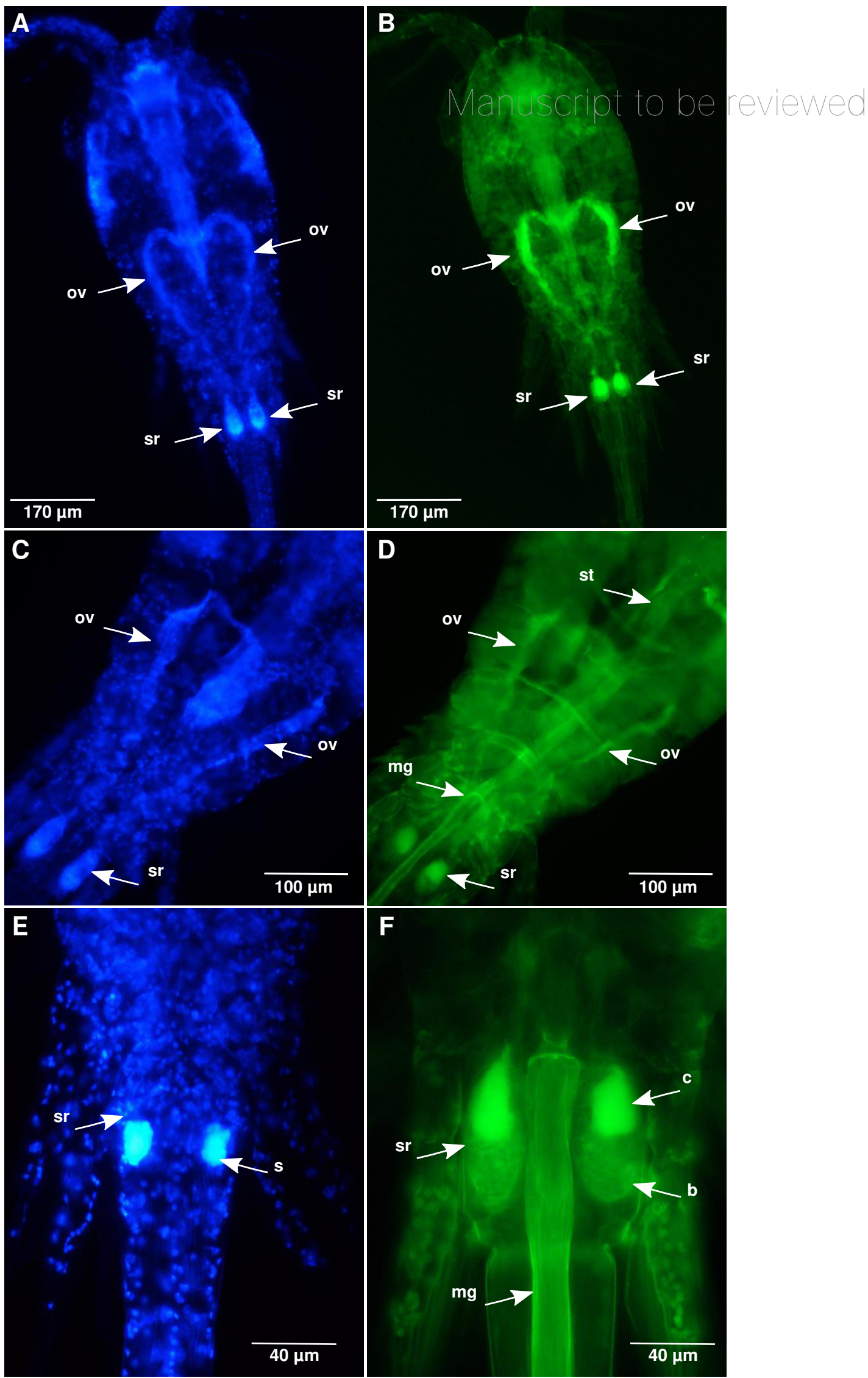
Figure 4 (on next page)

Oithona male reproductive system by DAPI and WGA-FITC fluorescence microscopy.

mg: midgut, fp: fecal pellet, sv: seminal vesicle, sp: spermiduct, a: heterogeneous chitin, b: diffuse chitin, c: chitin rich region. A. Dorsal view of the $O$.nana male reproductive system. B. Dorso-lateral view of the O.nana seminal vesicle. C. Lateral view of the male O.similis reproductive system. D. Lateral view of the O.nana male double sexual somite. E. Lateral view of the 0 .similis male double sexual somite. 


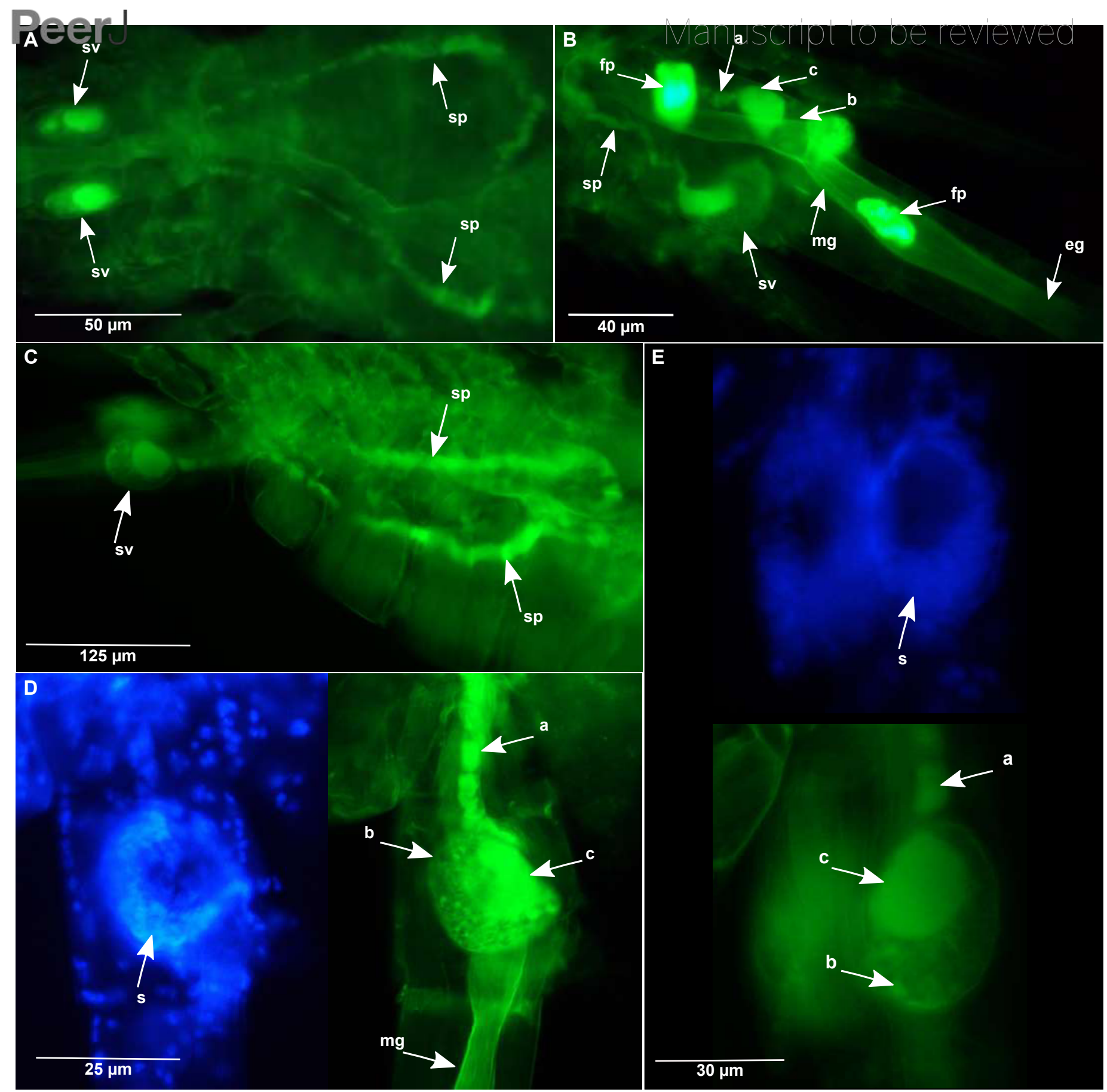


Figure $\mathbf{5}$ (on next page)

The diagram of the internal anatomy of a female Oithona nana.

st: stomach, mg: midgut, pm: peritrophic membrane, go: gonads, sh: shrinkages, fp: fecal pellet, hg: hindgut, sr: seminal receptacle, ov: oviduct. Thick black zones correspond to chitin rich areas. Dark gray zones correspond to heterogeneous chitin area. Light grey zones correspond to amorphous chitin areas. A. Diagram of the dorsal view of the digestive system. B. Diagram of the dorsal view of the reproductive system. 
A

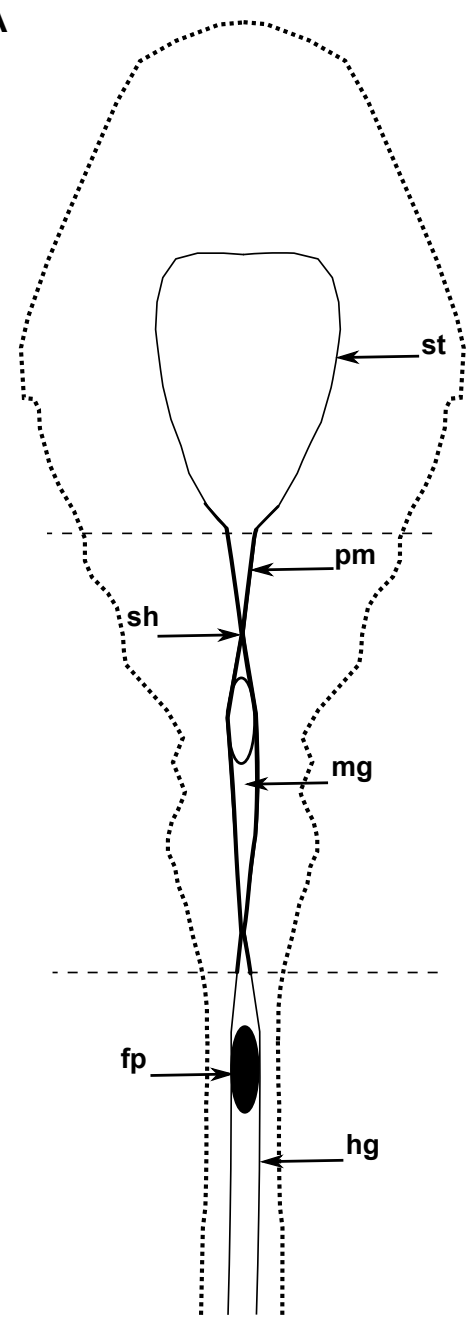

B

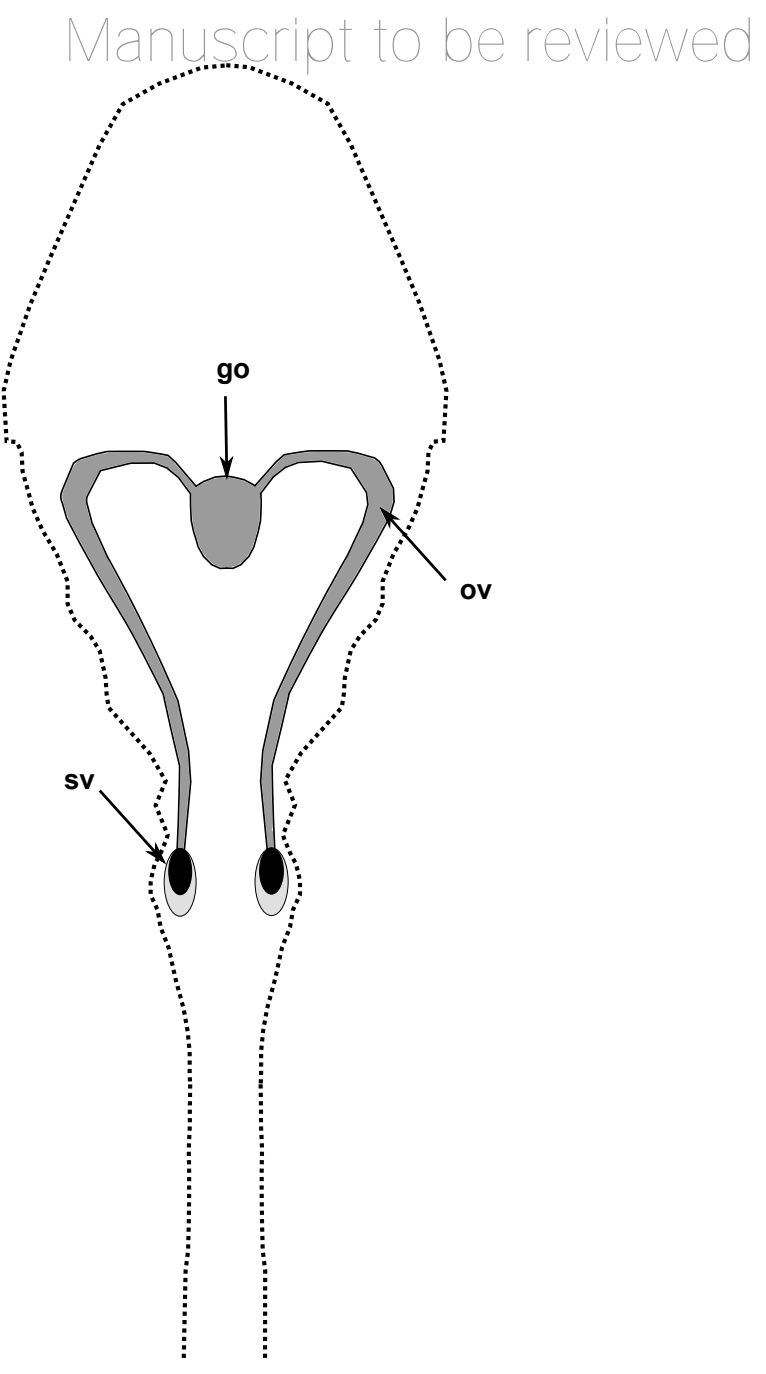

